Thorax 1985;40:351-357

\title{
A simple, inexpensive urine test of smoking
}

\author{
HEDLEY PEACH, GA ELLARD, PJ JENNER, RW MORRIS \\ From the Department of Community Medicine, St Thomas's Hospital Medical School, and the National \\ Institute for Medical Research, Mill Hill, London
}

\begin{abstract}
Three novel colorimetric methods of detecting urinary nicotine metabolites called the barbituric acid, diethylthiobarbituric acid (DETB), and DETB extraction methods were evaluated for use as a simple, cheap, objective test of smoking. Urine samples were collected from 103 male smokers and 78 male non-smokers working at two London factories. The smokers recorded the number of cigarettes smoked over the previous 36 hours. All three methods correctly classified the smokers. The DETB extraction method had a lower false positive rate (averaging $3 \%$ on morning and afternoon urine samples) than either the DETB or the barbituric acid method (12\% and $6 \%$ respectively) and was the best procedure for classifying subjects as "smokers" or "non-smokers." When a quantitative variant of the barbituric acid method was used there was a significant correlation $(\mathrm{r}=0.85, \mathrm{p}<0.001)$ between the ratios of urinary nicotine metabolites to creatinine and the number of cigarettes smoked. The ratios for smokers of $6-15,16-25$, and 26 or more cigarettes, however, overlapped considerably. The methods can be performed very rapidly and the reagent cost is equivalent to less than $1 \mathrm{p}$ per test.
\end{abstract}

Self reports of smoking behaviour need to be supplemented by an objective test of smoking. Large discrepancies have been found between self reported smoking status and objective tests of smoking. ${ }^{1-4}$ Although some non-smokers may have detectable levels of urinary nicotine due to passive smoking, ${ }^{5}$ the discrepancy has been largely attributed to deception on the part of smokers. ${ }^{2-4}$

Measurements of expired carbon monoxide, carboxyhaemoglobin, or thiocyanate in the blood, or nicotine in urine or saliva, have been used to validate self reported smoking habits. None of these tests, however, is really satisfactory for routine use by physicians - for example, in antismoking clinics. Even the measurement of expired carbon monoxide, which is the most acceptable to subjects, still requires the training and supervision of field workers in using expensive equipment. Measurement of urinary and salivary cotinine avoids the need for venepuncture, but the present method of assay using gas chromatography is expensive (about $£ 4$ a test), time consuming, and subject to interobserver variability. Analysis by radioimmunoassay does not

Address for reprint requests: Dr Hedley Peach, Department of Community Medicine, St Thomas's Hospital Medical School, London SE1 7EH.

Accepted 17 December 1984 confer any benefits in terms of the need for expensive equipment, cost (about $£ 8$ per test), or observer variability. A simpler, less expensive test of smoking would seem desirable.

In the past modifications of the König reaction have been used to measure urinary concentrations of isonicotinic acid and isonicotinylglycine, major metabolites of the antituberculosis drug isoniazid. ${ }^{6}$ Their sensitivity was such that they enabled a simple colorimetric urine test to be devised which was capable of detecting the ingestion of minute doses of isoniazid, thereby enabling it to be used as a marker to monitor patients' compliance..$^{7-9}$ Urine samples from non-smokers containing isonicotinic acid gave a characteristic blue colour when tested by the procedure. It was noticed, however, that urine samples from smokers not taking isoniazid gave distinctive orange colours and it was thought that nicotine and its metabolites were being detected. ${ }^{910}$ Preliminary studies showed that if diethylthiobarbituric acid was used in place of barbituric acid as the condensing agent in the König reaction a red product was obtained, which was readily extractable into ethyl acetate. These observations formed the basis of three simple potential urine tests to monitor nicotine intake.

The main purpose of this study was to establish the false positive and false negative rates of the urine tests in identifying smokers and non-smokers, 
so that they could be compared with the rates which have been published for the other methods. The interobserver variability as well as the variability of the test on duplicate urine samples was also determined.

\section{Methods}

\section{SUBJECTS}

Volunteers were recruited from men working in two London factories (Philips Electronics and Tate and Lyle Refineries Limited) and included 78 nonsmokers and 103 regular smokers of manufactured and hand rolled cigarettes. Pipe and cigar smokers were not enrolled.

\section{RECORDING OF SMOKING HABITS AND \\ COLLECTION OF URINE SAMPLES}

At the start of a working day the volunteers were each provided with two bottles for collecting urine and three randomly numbered tubes containing thymol, one marked "AM" and two "PM." The men were asked how many cigarettes they had already smoked that day and the brand of cigarette or tobacco used, and were requested to keep a record of every cigarette smoked over the next 36 hours, since cotinine, a major metabolite of nicotine, has a half life of about 20 hours. " As a check on the accuracy of recording, smokers of manufactured cigarettes were also asked to count the number of cigarettes in their packets at the start and end of each day, noting the number of new packets opened. All volunteers were asked whether they worked or lived with someone who smoked. The following day ( 24 hours after being given the bottles and tubes) the men were asked to provide one $5 \mathrm{ml}$ specimen of urine first thing in the morning and two $5 \mathrm{ml}$ specimens at a single voiding in the middle of the afternoon. The random numbering of the tubes and the removal of AM/PM labels before delivery to the laboratory ensured that the tests were carried out blind. The creatinine concentration of the two afternoon samples should have been identical and distinct from that of the morning sample and this was checked to ensure that the subjects had not mixed up the tubes when collecting the morning and afternoon specimens. Samples were preserved with a crystal of thymol and frozen at $-20^{\circ} \mathrm{C}$ before analysis. An investigation carried out on a random sample of the smoker's urine samples, however, showed that they could be stored for at least a week at room temperature without significant decomposition of either nicotine metabolites or creatinine.

To provide additional evidence on the ability of the methods to detect low concentrations of nicotine metabolites, such as cotinine, $0.05 \mathrm{ml}$ aliquots of aqueous cotinine with concentrations of $0,1,2,4,8$, and $16 \mu \mathrm{g} / \mathrm{ml}$ respectively were added to $0.45 \mathrm{ml}$ portions of each of the 24 afternoon urine samples from the non-exposed smokers. The samples were coded and randomised before being tested by the three methods and the results read by the two observers.

\section{LABORATORY METHODS}

The urine specimens were tested for the presence of nicotine metabolites by the three alternative qualitative procedures described below and classified by two observers (GAE and PJJ) with the naked eye. Where it was very difficult to make a decision the samples were classified as probably positive or probably negative; otherwise they were reported as definitely positive or definitely negative. No reference was made to any sort of comparison chart. The second observer had had no experience of using any of the methods before the study, while the first observer had only some experience of the direct barbituric acid method. Thorough mixing after each addition of reagent or solvent was achieved by agitation on a vortex mixer for about three seconds. Qualitative detection of nicotine metabolites: direct barbituric acid method Aliquots $(0.5 \mathrm{ml})$ of urine samples were pipetted into small test tubes with a 4 $\mathrm{mol} / \mathrm{l} \mathrm{pH} 4.7$ acetate buffer $(0.2 \mathrm{ml})$ and reacted by the sequential addition at 15 second intervals of freshly prepared $10 \%$ aqueous potassium cyanide $(0.1 \mathrm{ml}), 10 \%$ aqueous chloramine- $\mathrm{T}(0.1 \mathrm{ml})$, and $1 \%$ barbituric acid in acetone and water $(1: 1 \mathrm{v} / \mathrm{v})$ $(0.5 \mathrm{ml})$. A positive result was indicated by the appearance of an orange colour within 20 minutes. Qualitative detection of nicotine metabolites: direct diethylthiobarbituric acid (DETB) method This method is the same except that 1,3-diethyl-2thiobarbituric acid is used and the presence or absence of a pink-red colour is noted at 20 minutes. Qualitative detection of nicotine metabolites: diethylthiobarbituric acid (DETB) extraction method This method is the same except that the reaction product is extracted into $0.5 \mathrm{ml}$ ethyl acetate.

Quantitative determination of nicotine metabolites: barbituric acid method After all the factory specimens had been read by both observers with the naked eye as positive or negative for the presence of nicotine metabolites and the results recorded, urinary concentrations of nicotine metabolites were determined quantitatively. The optical densities of the specimens tested by the barbituric acid method were measured at $506 \mathrm{~nm} 20$ minutes after reaction and compared with the reading given by an aqueous solution of $10 \mu \mathrm{g} / \mathrm{ml}$ cotinine. Standard curves relating the optical density of the reaction product and cotinine concentration in aqueous solution and 
non-smoker's urine were prepared. Statistical evaluations showed that both curves were linear and parallel to each other. From the errors it was shown that the cotinine concentration could be predicted from the optical density with a residual standard deviation of only $0.08 \mu \mathrm{g} / \mathrm{ml}$. A cotinine standard was used since urinary concentrations of cotinine exceed those of nicotine when the $\mathrm{pH}$ of the urine is uncontrolled, ${ }^{12}$ as in this study.

Quantitative determination of nicotine metabolites: DETB extraction method The DETB extraction method was carried out with double volumes and after 20 minutes the reaction product was extracted into $2 \mathrm{ml}$ ethyl acetate. After centrifugation $1.5 \mathrm{ml}$ of the ethyl acetate extract was pipetted into a small test tube, $0.2 \mathrm{ml}$ ethanol was added, and the test tube was shaken. The optical density was then measured at $532 \mathrm{~nm}$ and nicotine metabolite concentrations were calculated by reference to the results obtained with an aqueous $10 \mu \mathrm{g} / \mathrm{ml}$ cotinine standard.

Determination of creatinine To allow for the effects of diuresis, ${ }^{12}$ ratios of nicotine metabolites to creatinine ( $\mu \mathrm{g}$ apparent cotinine/mg creatinine) were calculated after measurement of creatinine concentrations by a modification of the alkaline picrate method. ${ }^{13}$

\section{STATISTICAL METHODS}

The recordings of the two observers on the morning and first afternoon samples were used to calculate interobserver variability. The duplicate afternoon samples read by each observer were used to assess inter-test variability. The percentage agreement between two observers reading a series of nonidentical specimens or one observer reading a series of duplicate specimens as "positive" or "negative" can be high even when there is no real association between the readings. It was therefore necessary to calculate kappa statistics, which allow for this phenomenon, where values of kappa increasing from 0 to 1 indicate how much greater the agreement was than could be attributed to chance. ${ }^{14}$ The false positive and false negative rates of the three qualitative methods obtained by each observer in identifying smokers and non-smokers were calculated separately from the results obtained $(a)$ on the morning samples and $(b)$ on the afternoon samples.

\section{Results}

QUALITATIVE RESULTS

Table 1 shows the between observer variability of the three colorimetric methods in identifying smokers and non-smokers. The intertest variability as read by one observer is also shown in the table. The intertest variability was similar for the second observer. Although interobserver and intertest variability was low for all three methods, the intertest agreement was highest for the DETB extraction method.

Table 2 shows the false negative and false positive rates of the three methods in identifying smokers and non-smokers as read by one observer. For simplicity probable negatives have been included with the definite negatives and probable positives with the definite positives. All the urine samples from the smokers were found to be definitely positive by each of the three methods by both observers. Appreciable false positive rates were encountered but these were much lower for the DETB extraction method (morning and afternoon rates averaging 3\%) than for the direct barbituric acid and DETB procedures (morning and afternoon rates averaging 6\% and $12 \%$ respectively). Similar results were obtained by the second observer and the same specimens were nearly always misclassified by both observers. It was concluded that the DETB extraction method was the most satisfactory procedure for classifying subjects as "smokers" or "non-smokers."

After the qualitative tests had been completed and the results recorded, the false positive readings encountered among the non-smokers by each of the three methods were analysed according to their estimated excretion of nicotine metabolites and reported smoking exposures. The ratios of nicotine

Table 1 Interobserver and intertest variability of the colorimetric methods in determining smoking habit

\begin{tabular}{|c|c|c|c|c|}
\hline \multirow{2}{*}{$\begin{array}{l}\text { Type of } \\
\text { variability }\end{array}$} & \multicolumn{3}{|c|}{$\%$ of readings in agreement (kappa statistics* $(S E)$ ) } & \multirow[t]{2}{*}{$n$} \\
\hline & Barbituric acid method & DETB method & DETB extraction method & \\
\hline $\begin{array}{l}\text { Interobserver } \\
\text { Morning samples } \\
\text { Afternoon samples } \\
\text { Intertest }\end{array}$ & $\begin{array}{l}98(0.95(0.02)) \\
99(0.99(0.01))\end{array}$ & $\begin{array}{l}97(0.93(0.03)) \\
97(0.94(0.03))\end{array}$ & $\begin{array}{r}98(0.97(0.02)) \\
100(1.00(0.00))\end{array}$ & $\begin{array}{l}181 \\
179 \dagger\end{array}$ \\
\hline Afternoon samples & $94(0.87(0.04))$ & $93(0.85(0.04))$ & $99(0.98(0.02))$ & $174 \ddagger$ \\
\hline
\end{tabular}

See text.

†Two specimens missing or broken.

¥Seven specimens missing or broken

DETB-diethylthiobarbituric acid. 
Table 2 False positive and false negative rates of the three colorimetric methods for determining smoking habit

\begin{tabular}{|c|c|c|c|c|}
\hline \multirow[t]{2}{*}{ Method } & \multicolumn{2}{|c|}{ Morning sample } & \multicolumn{2}{|c|}{ Afternoon sample } \\
\hline & $\begin{array}{l}\text { False } \\
\text { negatives }\end{array}$ & $\begin{array}{l}\text { False } \\
\text { positives }\end{array}$ & $\begin{array}{l}\text { False } \\
\text { negatives }\end{array}$ & $\begin{array}{l}\text { False } \\
\text { positives }\end{array}$ \\
\hline $\begin{array}{l}\text { Barbituric acid } \\
\text { DETB } \\
\text { DETB extraction } \\
\text { True positives } \\
\text { True negatives } \\
\text { Total }\end{array}$ & $\begin{array}{l}0 \\
0 \\
0\end{array}$ & $\begin{array}{r}4(4 \%) \\
11(10 \%) \\
5(5 \%)\end{array}$ & $\begin{array}{l}0 \\
0 \\
0\end{array}$ & $\begin{array}{c}10(9 \%) \\
16(14 \%) \\
1(1 \%)\end{array}$ \\
\hline
\end{tabular}

*Two specimens missing or broken.

DETB-Diethylthiobarbituric acid.

metabolites to creatinine for all but one of the nonsmokers were very similar and averaged 0.7 (SD 0.2 ) and $0.9(0.2)$ for morning and afternoon specimens respectively. The exception was a subject with ratios of 2.0 and 2.7 who was exposed at home and at work. For this subject definitely positive results were obtained by each of the three qualitative methods. For the remaining 77 subjects about half of the false positives by the direct barbituric acid and DETB methods were classified as definitely positive, whereas all the false positives by the DETB extraction procedure were reported as only probably positive. Among these 77 non-smoking subjects there was no relationship between positivity and reported exposures to smoking at home or work or both. Most of the false positives occurred among highly coloured urine samples with raised creatinine concentrations.

The results obtained by one observer in the additional sensitivity study are summarised in table 3 . Similar results were obtained for the second observer. The findings suggest that a $50 \%$ positivity rate could be obtained with concentrations of about $0.45,0.35$, and $0.15 \mu \mathrm{g} / \mathrm{ml}$ continine for the barbituric acid, DETB, and DETB extraction methods respectively. This indicates that the extraction procedure is considerably more sensitive than the direct barbituric acid method for detecting nicotine metabolies in the urine.

QUANTITATIVE RESULTS

Figure 1 shows that with the direct barbituric acid method there was a strong correlation $(r=0.85)$, significantly different from zero $(p<0.001)$, between the ratios of nicotine metabolites to creatinine in the morning samples of urine on the second day and the number of cigarettes smoked on the first day. The correlation remained significant $(r=0.48$, $p<0.001)$ after exclusion of the non-smokers. Despite this, there was considerable overlap between the ranges of the ratios of nicotine metabolites to creatinine of smokers of 6-15, 16-25, and 26 or more cigarettes. Figure 2 shows the range of the ratios for the morning samples of urine for men grouped according to the number of cigarettes smoked on the previous day. Similar results were found for ratios of nicotine metabolites to creatinine in the afternoon samples of urine on the second day and the number of cigarettes smoked on that day as well as the total smoked over the first and second days. Investigations carried out on the afternoon samples showed that the quantitative variant of the DETB extraction procedure did not give a significantly better discrimination between the ratios of nicotine metabolites to creatinine in samples from smokers and non-smokers than did the simpler direct barbituric acid method.

\section{Discussion}

The reluctance of some people to admit to being smokers could create difficulties when trying to validate a new objective test of smoking. The greatest discrepancies between self reported smoking habit and objective tests of smoking have been reported among respondents from antismoking clinics ${ }^{4}$ and

Table 3 Sensitivity study: number of positive observations

\begin{tabular}{|c|c|c|c|c|c|c|c|}
\hline \multirow[t]{2}{*}{ Method } & \multicolumn{6}{|c|}{ Final cotinine concentration $(\mu \mathrm{g} / \mathrm{ml})$} & \multirow[t]{2}{*}{$n$} \\
\hline & 0 & 0.1 & 0.2 & 0.4 & 0.8 & 1.6 & \\
\hline $\begin{array}{l}\text { Barbituric acid } \\
\text { DETB } \\
\text { DETB extraction }\end{array}$ & $\begin{array}{l}1 \\
3 \\
1\end{array}$ & $\begin{array}{l}0 \\
3 \\
8\end{array}$ & $\begin{array}{r}1 \\
6 \\
20\end{array}$ & $\begin{array}{r}9 \\
14 \\
24\end{array}$ & $\begin{array}{l}18 \\
24 \\
24\end{array}$ & $\begin{array}{l}24 \\
24 \\
24\end{array}$ & $\begin{array}{l}24 \\
24 \\
24\end{array}$ \\
\hline
\end{tabular}

DETB-diethylthiobarituric acid. 


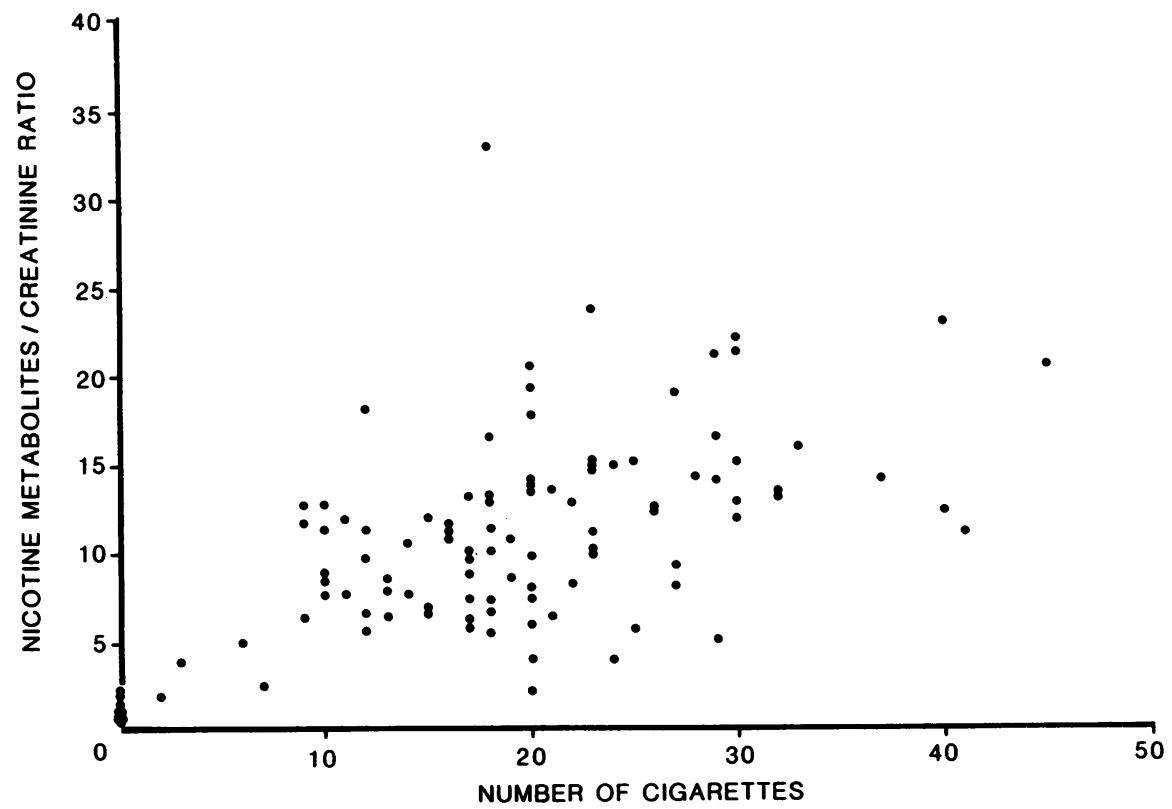

Fig 1 Relationship between ratios of nicotine metabolites to creatinine in morning urine samples on second day and number of cigarettes smoked on furst day ( $n=181)$.

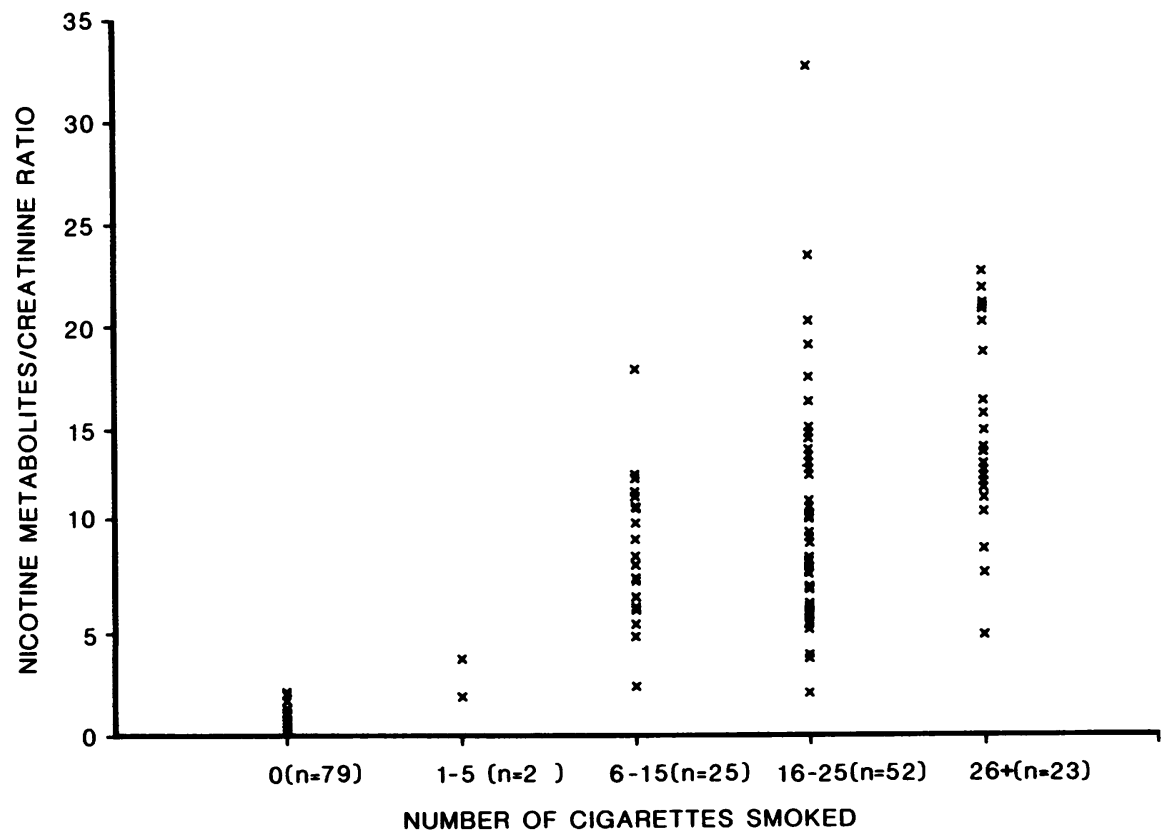

Fig 2 Ratios of nicotine metabolites to creatinine in morning urine samples for men smoking different numbers of cigarettes during the previous day $(n=181)$. 
patients given antismoking advice, ${ }^{23}$ where deception would be expected to be a problem. We chose a factory rather than a hospital population to reduce deception on the part of respondents. Prior knowledge that a test of smoking is to be carried out sometimes increases self reporting of smoking 1516 and respondents in this study were sent a letter explaining fully the purpose of the work. The men also kept a record of cigarettes smoked over the days of the study to eliminate recall errors.

With the DETB extraction method only four of the 78 non-smokers in our study were wrongly classified. The false negative and false positive rates for the DETB extraction method in identifying smokers and non-smokers-namely, zero and $3 \%$ respectively-compare very favourably with the published results of other tests. False negative rates of $12-34 \%$ have been found when expired carbon monoxide was used for identification, $5-17 \%$ with blood carboxyhaemoglobin, $7-36 \%$ with serum or plasma thiocyanate, $5 \%$ for plasma continine, and $28 \%$ with a combination of serum thiocyanate and expired carbon monoxide. ${ }^{3417-22}$ The corresponding proportions of false positives in these studies were $1-5 \%, 0-19 \%, 2 \%$, and $1 \%$ respectively.

It might be argued that considerable experience would be required to use this subjective qualitative procedure efficiently. We found, however, that the second observer, who was new to the procedures, performed as well as the more experienced observer. Furthermore, the proportion of false positives recorded among the first half of the samples analysed by the DETB extraction method was no higher than among the remainder for either observer. We may therefore conclude that the DETB extraction test is robust and likely to perform as well in the hands of other observers reading small numbers of specimens for the first time.

We had hoped that colorimetric estimation of the urinary concentration of nicotine metabolites might provide a useful measure of nicotine inhalation and might even be directly related to the number of cigarettes smoked. Although there was a significant overall correlation between the ratios of urinary nicotine metabolites to creatinine and number of cigarettes smoked (fig 1), the ratios for volunteers smoking $6-15,16-25$, and 26 or more cigarettes overlapped considerably. Such estimates of nicotine intake, however, correlated as closely with numbers of cigarettes smoked as other parameters used previously. Correlation coefficients of 0.4-0.5 have been found between serum thiocyanate, ${ }^{82122}$ carboxyhaemoglobin, ${ }^{22}$ expired carbon monoxide, ${ }^{21}$ and urinary nicotine ${ }^{s}$ concentrations and numbers of cigarettes smoked. In these studies the concentrations in light smokers and non-smokers also over- lapped considerably, as did those in moderate and heavy smokers. ${ }^{52122}$ It is now accepted that the intake of nicotine, cyanide, or carbon monoxide depends not only on the number of cigarettes smoked but also on the puffing and inhaling patterns. Consequently the number of cigarettes smoked accounts for only about $25 \%$ of the variance in urinary nicotine, expired carbon monoxide, and serum thiocyanate. ${ }^{22} 23$

If self reports of smoking need to be supplemented by an objective test of smoking it is an advantage if such a test is cheap, reasonably rapid, and capable of being applied to a large number of samples and if it does not require venepuncture or training of field workers in the use of expensive equipment. The DETB extraction procedure meets these criteria. It requires only urine samples, is simple to perform, and takes less than one minute a test on average, and reagent costs are less than $1 p$ a specimen. Furthermore, the quantitative barbituric acid ratio method for estimating nicotine intake is also cheap and rapid to perform (about three minutes a sample) and both methods require only equipment which should be available in any biochemistry laboratory.

We would like to thank Professor WW Holland for encouraging us to do the study, Miss D Shah for assistance with computing, and Mr DR Ellard for help with the chemical analyses. We are indebted to Dr Terry and Sister Saku of Philips Electronics and Dr Biss and Sister Wilkinson of Tate and Lyle Thameside Refineries for their invaluable help in setting up the study. We thank Mrs Linda Clarke for word processing the manuscript and Ms V Norcott Martin of the department of medical illustration, Rayne Institute, St Thomas's Hospital, for drawing the figures. The study was undertakin in connection with research being funded by the Tobacco Products Research Trust.

\section{References}

1 Gillies PA, Wilcox B, Coates C, Kristmundsdottir F, Reid DJ. Use of objective measurement in the validation of self reported smoking in children aged 10-11 years: saliva thiocyanate. J Epidemiol Commun Health 1982;36:205-8.

2 Wilcox RG, Hughes J, Roland J. Verification of smoking history on patients after infarction using urinary nicotine and cotinine measurements. $\mathrm{Br} \mathrm{Med} J$ 1979;ii:1026-8.

3 Sillett RW, Wilson MB, Malcolm RE, Ball KP. Deception among smokers. Br Med J 1978; ii:1185-6.

4 Ohlin $\mathrm{P}$, Lundh $\mathrm{B}$, Westling $\mathrm{H}$. Carbon monoxide levels and reported cessation of smoking. Psychopharmacology 1976;49:263-5. 
5 Feyerabend C, Higenbottam T, Russell MAH. Nicotine concentrations in urine and saliva of smokers and non-smokers. $\mathrm{Br}$ Med J 1982;284:1002-4.

6 Ellard GA, Gammon PT, Wallace SM. The determination of isoniazid and its metabolites acetylisoniazid, monoacetyl hydrazine, diacetylhydrazine, isonicotinic acid and isonicotinylglycine in serum and urine. Biochem J 1972;126:449-58.

7 Ellard GA, Greenfield C. A sensitive urine-test method for monitoring the ingestion of isoniazid. J Clin Pathol 1977;30:84-7.

8 Ellard GA, Jenner PJ, Downs PA. An evaluation of the potential use of isoniazid, acetylisoniazid and isonicotinic acid for monitoring the self-administration of drugs. Br J Clin Pharmacol 1980;10:369-81.

9 Stanley JNA, Pearson JMH, Ellard GA. An investigation of dapsone compliance using an isoniazid-marked formulation. Leprosy Reveiw 1983;54:317-25.

10 Bowman ER, Turnbull LB, McKennis H. Metabolism of nicotine in the human and excretion of pyridine compounds by smokers. J Pharmacol Exp Ther 1959;127:92-5.

11 Benowitz ML, Kuyt F, Jacob P, Jones RT, Osman A. Cotinine disposition and effects. Clin Pharmacol Ther 1983;34:604-11.

12 Langone JJ, Gjika HB, Vunakis H Van. Nicotine and its metabolites. Radioimmunoassays for nicotine and cotinine. Biochemistry 1973;12:5025-30.

13 Ellard GA, Gammon PT, Helmy HB, Rees RJW. Urine test to monitor the self-administration of dapsone by leprosy patients. Am J Trop Med Hyg 1974;23:404-7.

14 Fleiss JL. Statistical methods for rates and proportions. 2nd ed. New York: Wiley, 1981:217-21.
15 Evans RI, Hansen WB, Mittelmark MB. Increasing the validity of self reports of smoking behaviour in children. J Appl Psychol 1977;62:521-3.

16 Bauman KE, Dent CW. Influence of an objective measure on self-reports of behaviour. J Appl Psychol 1982; 67:623-8.

17 Petitti DB, Friedman GD, Kahn W. Accuracy of information on smoking habits on self-administration research questionnaires. Am J Publ Health 1981;71:308-11.

18 Kornitzer M, Vanhemeldonck A, Bourdoux P, Backer GD. Belgian heart disease prevention project: comparison of self-reported smoking behaviour with serum thiocyanate concentrations. J Epidemiol Commun Health 1983;37:132-6.

19 Cohen JD, Bartsch GE. A comparison between carboxyhaemoglobin and serum thiocyanate determinations as indicators of cigarette smoking. Am J Publ Health 1980;70:284-6.

20 Williams CL, Eng A, Botvin GJ, Hill P, Wynder EL. Validation of students' self-reported cigarette smoking status with plasma continine levels. Am J Publ Health 1979;69:1272-4.

21 Vogt TM, Selvin S, Widdowson S, Hulley SB. Expired air carbon monoxide and serum thiocyanate and objective measures of cigarette exposure. Am J Publ Health 1977;67:545-9.

22 Vesey CJ, Saloojee Y, Cole PV, Russel MAH. Blood carboxy haemoglobin, plasma thiocyanate, and cigarette consumption implications for epidemiological studies in smokers. Br Med J 1982;284:1516-8.

23 Vogt TM, Selvin S, Hulley SB. Comparison of biochemical and questionnaire estimates of tobacco exposure. Prev Med 1979;8:23-33. 